\title{
AN ASYMPTOTIC RESULT FOR SOME DELAY DIFFERENCE EQUATIONS WITH CONTINUOUS VARIABLE
}

\author{
CH. G. PHILOS AND I. K. PURNARAS
}

Received 14 October 2003

We consider a nonhomogeneous linear delay difference equation with continuous variable and establish an asymptotic result for the solutions. Our result is obtained by the use of a positive root with an appropriate property of the so-called characteristic equation of the corresponding homogeneous linear (autonomous) delay difference equation. More precisely, we show that, for any solution, the limit of a specific integral transformation of it, which depends on a suitable positive root of the characteristic equation, exists as a real number and it is given explicitly in terms of the positive root of the characteristic equation and the initial function.

\section{Introduction and statement of the main result}

Difference equations with continuous variable are difference equations in which the unknown function is a function of a continuous variable. (The term "difference equation" is usually used for difference equations with discrete variables.) In practice, time is often involved as the independent variable in difference equations with continuous variable. In view of this fact, we may also refer to them as difference equations with continuous time. Difference equations with continuous variable appear as natural descriptions of observed evolution phenomena in many branches of the natural sciences (see, e.g., the book by Sharkovsky et al. [15]; see, also, the paper by Ladas [9]). The book [15] presents an exposition of unusual properties of difference equations (and, in particular, of difference equations with continuous variable). For some results on the oscillation of difference equations with continuous variable, we choose to refer to Domshlak [1], Ladas et al. [10], Shen [16], Yan and Zhang [17], and Zhang et al. [18] (and the references cited therein).

Driver et al. [4] obtained some significant results on the asymptotic behavior, the nonoscillation, and the stability of the solutions of first-order scalar linear delay differential equations with constant coefficients and one constant delay. See Driver [2] for some similar important results for first-order scalar linear delay differential equations with infinitely many distributed delays. Several extensions of the results in [4] for delay differential equations as well as for neutral delay differential equations have been presented by 
Philos [11], Kordonis et al. [6], and Philos and Purnaras [12]. For some related results, we refer to Graef and Qian [5]. Moreover, the discrete analogues of the results in $[6,11]$ have been given by Kordonis and Philos [7] and Kordonis et al. [8], respectively. The results in $[7,8]$ concern difference equations with discrete variable. For some related results for difference equations (with discrete variable), see Driver et al. [3] and Pituk [13, 14]. Motivated by the results in [4] as well as by those in the above-mentioned papers, we here make a first attempt to arrive at analogous results for the case of difference equations with continuous variable.

In this paper, we give an asymptotic criterion for the solutions of some linear delay difference equations with continuous variable.

Consider the delay difference equation with continuous variable

$$
x(t)-x(t-\sigma)=a x(t-\sigma)+\sum_{j=1}^{k} b_{j} x\left(t-\tau_{j}\right)+f(t),
$$

where $k$ is a positive integer, $a$ and $b_{j} \neq 0(j=1, \ldots, k)$ are real constants, $\sigma$ and $\tau_{j}(j=$ $1, \ldots, k)$ are positive real numbers with $\tau_{j_{1}} \neq \tau_{j_{2}}\left(j_{1}, j_{2}=1, \ldots, k ; j_{1} \neq j_{2}\right)$ such that $\tau_{j}>\sigma$ $(j=1, \ldots, k)$, and $f$ is a continuous real-valued function on the interval $[0, \infty)$.

We define

$$
\tau=\max _{j=1, \ldots, k} \tau_{j}
$$

( $\tau$ is a positive real number with $\tau>\sigma$ ).

By a solution of the difference equation (1.1), we mean a continuous real-valued function $x$ defined on the interval $[-\tau, \infty)$ which satisfies (1.1) for all $t \geq 0$.

In the sequel, by $\Phi$ we will denote the set of all continuous real-valued functions $\phi$ defined on the interval $[-\tau, 0]$, which satisfy the "compatibility condition"

$$
\phi(0)-\phi(-\sigma)=a \phi(-\sigma)+\sum_{j=1}^{k} b_{j} \phi\left(-\tau_{j}\right)+f(0) .
$$

By the method of steps, one can easily see that, for any given initial function $\phi \in \Phi$, there exists a unique solution $x$ of the delay difference equation (1.1) which satisfies the initial condition

$$
x(t)=\phi(t) \quad \text { for } t \in[-\tau, 0]
$$

this function $x$ will be called the solution of the initial problem (1.1), (1.2), (1.3), and (1.4) or, more briefly, the solution of (1.1), (1.2), (1.3), and (1.4).

In the case where the function $f$ is identically zero on the interval $[0, \infty)$, the delay difference equation (1.1) reduces to

$$
x(t)-x(t-\sigma)=a x(t-\sigma)+\sum_{j=1}^{k} b_{j} x\left(t-\tau_{j}\right) .
$$

Furthermore, we introduce the following assumption. 
(H) There exist integers $m_{j}>1(j=1, \ldots, k)$ such that

$$
\tau_{j}=m_{j} \sigma \quad(j=1, \ldots, k) .
$$

Throughout the paper, it will be supposed that assumption $(\mathrm{H})$ holds without any further mention.

If we look for solutions of (1.5) of the form $x(t)=\lambda^{t / \sigma}$ for $t \geq-\tau$, then we can easily see that $\lambda$ satisfies

$$
\lambda-1=a+\sum_{j=1}^{k} b_{j} \lambda^{-m_{j}+1}
$$

Equation (1.7) will be called the characteristic equation of the delay difference equation (1.5).

To obtain the main result of the paper, we will make use of a positive root $\lambda_{0}$ of the characteristic equation (1.7) with the property

$$
\sum_{j=1}^{k}\left|b_{j}\right|\left(m_{j}-1\right) \lambda_{0}^{-m_{j}}<1
$$

The following lemma due to Kordonis et al. [8] provides sufficient conditions for the characteristic equation (1.7) to have a positive root $\lambda_{0}$ with the property (1.8).

Lemma 1.1. Set

$$
m=\max _{j=1, \ldots, k} m_{j}
$$

and assume that

$$
\sum_{j=1}^{k} b_{j} \frac{m^{m_{j}}}{(m-1)^{m_{j}-1}}>-1-a m, \quad \sum_{j=1}^{k}\left|b_{j}\right| \frac{m_{j}-1}{m-1} \cdot \frac{m^{m_{j}}}{(m-1)^{m_{j}-1}} \leq 1 .
$$

Then, in the interval $((m-1) / m, \infty)$, the characteristic equation (1.7) has a unique (positive) root $\lambda_{0}$; this root has the property (1.8).

For some comments on the conditions imposed in the above lemma, we refer to [8]. Moreover, we notice that a generalization of this lemma has been given by Kordonis and Philos [7].

Our main result is the following theorem.

THeOREM 1.2. Let $\lambda_{0}$ be a positive root of the characteristic equation (1.7) with the property (1.8) and assume that

$$
F_{\lambda_{0}} \equiv \int_{0}^{\infty} \lambda_{0}^{-t / \sigma} f(t) d t
$$

exists (as a real number). 
4 Difference equations with continuous variable

Then, for any $\phi \in \Phi$, the solution $x$ of (1.1), (1.2), (1.3), and (1.4) satisfies

$$
\lim _{t \rightarrow \infty} \int_{t-\sigma}^{t} \lambda_{0}^{-s / \sigma} x(s) d s=\frac{L_{\lambda_{0}}(\phi)+F_{\lambda_{0}}}{1+\sum_{j=1}^{k} b_{j}\left(m_{j}-1\right) \lambda_{0}^{-m_{j}}}
$$

where

$$
L_{\lambda_{0}}(\phi)=\int_{-\sigma}^{0} \lambda_{0}^{-s / \sigma} \phi(s) d s+\sum_{j=1}^{k} b_{j} \lambda_{0}^{-m_{j}} \int_{-\tau_{j}}^{-\sigma} \lambda_{0}^{-s / \sigma} \phi(s) d s .
$$

Note. Property (1.8) guarantees that

$$
1+\sum_{j=1}^{k} b_{j}\left(m_{j}-1\right) \lambda_{0}^{-m_{j}}>0
$$

Clearly, our theorem can be applied to the delay difference equation (1.5) with $F_{\lambda_{0}}=0$.

We can immediately see that $\lambda_{0}=1$ is a (positive) root of the characteristic equation (1.7) with the property (1.8) if and only if

$$
a+\sum_{j=1}^{k} b_{j}=0, \quad \sum_{j=1}^{k}\left|b_{j}\right|\left(m_{j}-1\right)<1 .
$$

Thus, an application of our theorem with $\lambda_{0}=1$ leads to the following result.

Let condition (1.15) be satisfied and assume that $\int_{0}^{\infty} f(t) d t$ exists (as a real number). Then, for any $\phi \in \Phi$, the solution $x$ of (1.1), (1.2), (1.3), and (1.4) satisfies

$$
\lim _{t \rightarrow \infty} \int_{t-\sigma}^{t} x(s) d s=\frac{\left[\int_{-\sigma}^{0} \phi(s) d s+\sum_{j=1}^{k} b_{j} \int_{-\tau_{j}}^{-\sigma} \phi(s) d s\right]+\int_{0}^{\infty} f(s) d s}{1+\sum_{j=1}^{k} b_{j}\left(m_{j}-1\right)} .
$$

Note. The second assumption of (1.15) guarantees that

$$
1+\sum_{j=1}^{k} b_{j}\left(m_{j}-1\right)>0
$$

\section{Proof of Theorem $\mathbf{1 . 2}$}

First of all, we define

$$
\mu_{\lambda_{0}}=\sum_{j=1}^{k}\left|b_{j}\right|\left(m_{j}-1\right) \lambda_{0}^{-m_{j}}, \quad \gamma_{\lambda_{0}}=\sum_{j=1}^{k} b_{j}\left(m_{j}-1\right) \lambda_{0}^{-m_{j}}
$$

Property (1.8) means that

$$
0<\mu_{\lambda_{0}}<1
$$


Furthermore, we have $\left|\gamma_{\lambda_{0}}\right| \leq \mu_{\lambda_{0}}<1$. This, in particular, implies that

$$
1+\gamma_{\lambda_{0}}>0
$$

Consider an arbitrary function $\phi \in \Phi$ and let $x$ be the solution of (1.1), (1.2), (1.3), and (1.4). We will show that

$$
\lim _{t \rightarrow \infty} \int_{t-\sigma}^{t} \lambda_{0}^{-s / \sigma} x(s) d s=\frac{L_{\lambda_{0}}(\phi)+F_{\lambda_{0}}}{1+\gamma_{\lambda_{0}}}
$$

Set

$$
y(t)=\lambda_{0}^{-t / \sigma} x(t) \quad \text { for } t \geq-\tau .
$$

Then, by taking into account the fact that $\tau_{j}=m_{j} \sigma(j=1, \ldots, k)$ and using the hypothesis that $\lambda_{0}$ is a (positive) root of the characteristic equation (1.7), we obtain, for every $t \geq 0$,

$$
\begin{aligned}
x(t) & -x(t-\sigma)-a x(t-\sigma)-\sum_{j=1}^{k} b_{j} x\left(t-\tau_{j}\right)-f(t) \\
& =\lambda_{0}^{t / \sigma}\left[y(t)-\lambda_{0}^{-1} y(t-\sigma)-a \lambda_{0}^{-1} y(t-\sigma)-\sum_{j=1}^{k} b_{j} \lambda_{0}^{-\tau_{j} / \sigma} y\left(t-\tau_{j}\right)\right]-f(t) \\
& =\lambda_{0}^{t / \sigma}\left[y(t)-\lambda_{0}^{-1}(1+a) y(t-\sigma)-\sum_{j=1}^{k} b_{j} \lambda_{0}^{-m_{j}} y\left(t-\tau_{j}\right)\right]-f(t) \\
& =\lambda_{0}^{t / \sigma}\left[y(t)-\lambda_{0}^{-1}\left(\lambda_{0}-\sum_{j=1}^{k} b_{j} \lambda_{0}^{-m_{j}+1}\right) y(t-\sigma)-\sum_{j=1}^{k} b_{j} \lambda_{0}^{-m_{j}} y\left(t-\tau_{j}\right)\right]-f(t) \\
& =\lambda_{0}^{t / \sigma}\left[y(t)-y(t-\sigma)+\left(\sum_{j=1}^{k} b_{j} \lambda_{0}^{-m_{j}}\right) y(t-\sigma)-\sum_{j=1}^{k} b_{j} \lambda_{0}^{-m_{j}} y\left(t-\tau_{j}\right)\right]-f(t) .
\end{aligned}
$$

So, the fact that $x$ satisfies (1.1) for $t \geq 0$ is equivalent to the fact that $y$ satisfies

$$
y(t)-y(t-\sigma)=-\sum_{j=1}^{k} b_{j} \lambda_{0}^{-m_{j}}\left[y(t-\sigma)-y\left(t-\tau_{j}\right)\right]+\lambda_{0}^{-t / \sigma} f(t) \quad \text { for } t \geq 0 .
$$

On the other hand, the initial condition (1.4) reduces to

$$
y(t)=\lambda_{0}^{-t / \sigma} \phi(t) \quad \text { for } t \in[-\tau, 0] .
$$

Furthermore, because of our assumption on the function $f$, it is clear that (2.7) can equivalently be written as follows:

$$
\frac{d}{d t}\left[\int_{t-\sigma}^{t} y(s) d s\right]=\frac{d}{d t}\left[-\sum_{j=1}^{k} b_{j} \lambda_{0}^{-m_{j}} \int_{t-\tau_{j}}^{t-\sigma} y(s) d s-\int_{t}^{\infty} \lambda_{0}^{-s / \sigma} f(s) d s\right] \quad \text { for } t \geq 0 .
$$


6 Difference equations with continuous variable

Moreover, by using (2.8) and taking into account the definitions of $L_{\lambda_{0}}(\phi)$ and $F_{\lambda_{0}}$, we get

$$
\begin{aligned}
\left.\left\{\int_{t-\sigma}^{t} y(s) d s-\left[-\sum_{j=1}^{k} b_{j} \lambda_{0}^{-m_{j}} \int_{t-\tau_{j}}^{t-\sigma} y(s) d s-\int_{t}^{\infty} \lambda_{0}^{-s / \sigma} f(s) d s\right]\right\}\right|_{t=0} \\
\quad=\int_{-\sigma}^{0} y(s) d s+\sum_{j=1}^{k} b_{j} \lambda_{0}^{-m_{j}} \int_{-\tau_{j}}^{-\sigma} y(s) d s+\int_{0}^{\infty} \lambda_{0}^{-s / \sigma} f(s) d s \\
=\left[\int_{-\sigma}^{0} \lambda_{0}^{-s / \sigma} \phi(s) d s+\sum_{j=1}^{k} b_{j} \lambda_{0}^{-m_{j}} \int_{-\tau_{j}}^{-\sigma} \lambda_{0}^{-s / \sigma} \phi(s) d s\right]+\int_{0}^{\infty} \lambda_{0}^{-s / \sigma} f(s) d s \\
=L_{\lambda_{0}}(\phi)+F_{\lambda_{0}} .
\end{aligned}
$$

Thus, (2.7) is equivalent to

$$
\int_{t-\sigma}^{t} y(s) d s=-\sum_{j=1}^{k} b_{j} \lambda_{0}^{-m_{j}} \int_{t-\tau_{j}}^{t-\sigma} y(s) d s-\int_{t}^{\infty} \lambda_{0}^{-s / \sigma} f(s) d s+\left[L_{\lambda_{0}}(\phi)+F_{\lambda_{0}}\right] \quad \text { for } t \geq 0 .
$$

Next, we define

$$
Y(t)=\int_{t-\sigma}^{t} y(s) d s \quad \text { for } t \geq-\tau+\sigma
$$

Then, by taking into account the fact that $\tau_{j}=m_{j} \sigma(j=1, \ldots, k)$, we have, for any $j \in$ $\{1, \ldots, k\}$ and every $t \geq 0$,

$$
\begin{aligned}
\int_{t-\tau_{j}}^{t-\sigma} y(s) d s & =\int_{t-m_{j} \sigma}^{t-\sigma} y(s) d s=\sum_{i=1}^{m_{j}-1} \int_{t-(i+1) \sigma}^{t-i \sigma} y(s) d s \\
& =\sum_{i=1}^{m_{j}-1} \int_{(t-i \sigma)-\sigma}^{(t-i \sigma)} y(s) d s=\sum_{i=1}^{m_{j}-1} Y(t-i \sigma) .
\end{aligned}
$$

Hence, (2.11) takes the following equivalent form:

$$
Y(t)=-\sum_{j=1}^{k} b_{j} \lambda_{0}^{-m_{j}}\left[\sum_{i=1}^{m_{j}-1} Y(t-i \sigma)\right]-\int_{t}^{\infty} \lambda_{0}^{-s / \sigma} f(s) d s+\left[L_{\lambda_{0}}(\phi)+F_{\lambda_{0}}\right] \quad \text { for } t \geq 0
$$

Also, (2.8) becomes

$$
Y(t)=\int_{t-\sigma}^{t} \lambda_{0}^{-s / \sigma} \phi(s) d s \quad \text { for } t \in[-\tau+\sigma, 0] .
$$

Now, we introduce the function

$$
z(t)=Y(t)-\frac{L_{\lambda_{0}}(\phi)+F_{\lambda_{0}}}{1+\gamma_{\lambda_{0}}} \quad \text { for } t \geq-\tau+\sigma
$$


By using the way of the definition of $\gamma_{\lambda_{0}}$, one can easily see that (2.14) reduces to the following equivalent equation:

$$
z(t)=-\sum_{j=1}^{k} b_{j} \lambda_{0}^{-m_{j}}\left[\sum_{i=1}^{m_{j}-1} z(t-i \sigma)\right]-\int_{t}^{\infty} \lambda_{0}^{-s / \sigma} f(s) d s \quad \text { for } t \geq 0 .
$$

On the other hand, (2.15) can equivalently be written as

$$
z(t)=\int_{t-\sigma}^{t} \lambda_{0}^{-s / \sigma} \phi(s) d s-\frac{L_{\lambda_{0}}(\phi)+F_{\lambda_{0}}}{1+\gamma_{\lambda_{0}}} \quad \text { for } t \in[-\tau+\sigma, 0] .
$$

Thus, $z$ is a solution of the delay difference equation (2.17) which satisfies the initial condition (2.18), that is, $z$ is a solution of the initial problem (2.17) and (2.18).

By the definitions of $y, Y$, and $z$, we immediately see that (2.4) is equivalent to

$$
\lim _{t \rightarrow \infty} z(t)=0
$$

So, the proof of the theorem can be completed by showing (2.19).

Since $0<\mu_{\lambda_{0}}<1$, we can consider a number $\epsilon_{0} \in(0,1)$ so that

$$
0<\mu_{\lambda_{0}}+\epsilon_{0}<1
$$

Furthermore, by using our assumption on the function $f$, we can inductively define a sequence of points $\left(t_{n}\right)_{n \geq 1}$ in $[0, \infty)$ with

$$
t_{n+1}-t_{n} \geq \tau-\sigma \quad(n=1,2, \ldots)
$$

such that, for all $n=1,2, \ldots$,

$$
\left|\int_{t}^{\infty} \lambda_{0}^{-s / \sigma} f(s) d s\right| \leq \epsilon_{0}\left(\mu_{\lambda_{0}}+\epsilon_{0}\right)^{n-1} \quad \text { for every } t \geq t_{n}
$$

Set $t_{0}=-\tau+\sigma$ and

$$
M=\max \left\{1, \max _{t \in\left[t_{0}, t_{1}\right]}|z(t)|\right\} .
$$

Then $M \geq 1$ and

$$
|z(t)| \leq M \quad \text { for } t \in\left[t_{0}, t_{1}\right]
$$

We will prove that $M$ is a bound of $z$ on the whole interval $\left[t_{0}, \infty\right)$, that is,

$$
|z(t)| \leq M \quad \forall t \geq t_{0} .
$$

To this end, we consider an arbitrary number $\epsilon>0$. We claim that

$$
|z(t)|<M+\epsilon \quad \text { for every } t \geq t_{0}
$$


8 Difference equations with continuous variable

Otherwise, in view of (2.24), there exists a point $t^{*}>t_{1}$ so that

$$
|z(t)|<M+\epsilon \quad \text { for } t \in\left[t_{0}, t^{*}\right), \quad\left|z\left(t^{*}\right)\right|=M+\epsilon .
$$

Then, by using (2.22) with $n=1$, from (2.17), we obtain

$$
\begin{aligned}
M+\epsilon & =\left|z\left(t^{*}\right)\right| \leq \sum_{j=1}^{k}\left|b_{j}\right| \lambda_{0}^{-m_{j}}\left[\sum_{i=1}^{m_{j}-1}\left|z\left(t^{*}-i \sigma\right)\right|\right]+\left|\int_{t^{*}}^{\infty} \lambda_{0}^{-s / \sigma} f(s) d s\right| \\
& <\left[\sum_{j=1}^{k}\left|b_{j}\right|\left(m_{j}-1\right) \lambda_{0}^{-m_{j}}\right](M+\epsilon)+\epsilon_{0},
\end{aligned}
$$

and consequently, in view of the definition of $\mu_{\lambda_{0}}$ and the fact that $M \geq 1$ and $0<\mu_{\lambda_{0}}+$ $\epsilon_{0}<1$, we have

$$
\begin{aligned}
M+\epsilon & <\mu_{\lambda_{0}}(M+\epsilon)+\epsilon_{0}<\mu_{\lambda_{0}}(M+\epsilon)+\epsilon_{0}(M+\epsilon) \\
& =\left(\mu_{\lambda_{0}}+\epsilon_{0}\right)(M+\epsilon)<M+\epsilon .
\end{aligned}
$$

This is a contradiction and hence (2.26) holds true. From the fact that (2.26) is fulfilled for all numbers $\epsilon>0$, it follows immediately that (2.25) is always satisfied. Next, by using (2.22) (with $n=1$ ) and (2.25), and taking into account the way of the definition of $\mu_{\lambda_{0}}$ and the fact that $M \geq 1$, from (2.17), we get, for every $t \geq t_{1}$,

$$
\begin{aligned}
|z(t)| & \leq \sum_{j=1}^{k}\left|b_{j}\right| \lambda_{0}^{-m_{j}}\left[\sum_{i=1}^{m_{j}-1}|z(t-i \sigma)|\right]+\left|\int_{t}^{\infty} \lambda_{0}^{-s / \sigma} f(s) d s\right| \\
& \leq\left[\sum_{j=1}^{k}\left|b_{j}\right|\left(m_{j}-1\right) \lambda_{0}^{-m_{j}}\right] M+\epsilon_{0} \\
& =\mu_{\lambda_{0}} M+\epsilon_{0} \\
& \leq \mu \lambda_{0} M+\epsilon_{0} M .
\end{aligned}
$$

Therefore,

$$
|z(t)| \leq\left(\mu_{\lambda_{0}}+\epsilon_{0}\right) M \quad \text { for all } t \geq t_{1}
$$

Our purpose is to show that for each $n=0,1,2, \ldots$,

$$
|z(t)| \leq\left(\mu_{\lambda_{0}}+\epsilon_{0}\right)^{n} M \quad \forall t \geq t_{n}
$$

We observe that (2.32) with $n=0$ coincides with (2.25), while (2.32) with $n=1$ is the same as (2.31). Assume that (2.32) is true for $n=v$, where $v$ is a positive integer, that is,

$$
|z(t)| \leq\left(\mu_{\lambda_{0}}+\epsilon_{0}\right)^{\nu} M \quad \forall t \geq t_{\nu}
$$


Then, in view of (2.22) (with $n=\nu+1)$ and (2.33) as well as of the definition of $\mu_{\lambda_{0}}$ and the fact that $M \geq 1$, from (2.17), it follows that, for $t \geq t_{\nu+1}$,

$$
\begin{aligned}
|z(t)| & \leq \sum_{j=1}^{k}\left|b_{j}\right| \lambda_{0}^{-m_{j}}\left[\sum_{i=1}^{m_{j}-1}|z(t-i \sigma)|\right]+\left|\int_{t}^{\infty} \lambda_{0}^{-s / \sigma} f(s) d s\right| \\
& \leq\left[\sum_{j=1}^{k}\left|b_{j}\right|\left(m_{j}-1\right) \lambda_{0}^{-m_{j}}\right]\left(\mu_{\lambda_{0}}+\epsilon_{0}\right)^{v} M+\epsilon_{0}\left(\mu_{\lambda_{0}}+\epsilon_{0}\right)^{v} \\
& =\mu_{\lambda_{0}}\left(\mu_{\lambda_{0}}+\epsilon_{0}\right)^{v} M+\epsilon_{0}\left(\mu_{\lambda_{0}}+\epsilon_{0}\right)^{v} \\
& \leq \mu \lambda_{0}\left(\mu \lambda_{0}+\epsilon_{0}\right)^{v} M+\epsilon_{0}\left(\mu \lambda_{0}+\epsilon_{0}\right)^{v} M \\
& =\left(\mu_{\lambda_{0}}+\epsilon_{0}\right)^{\nu+1} M .
\end{aligned}
$$

Thus, (2.32) is also true for $n=v+1$. Hence, by the induction principle, we conclude that (2.32) holds true for all nonnegative integers $n$. Finally, since $0<\mu_{\lambda_{0}}+\epsilon_{0}<1$, we have

$$
\lim _{n \rightarrow \infty}\left(\mu_{\lambda_{0}}+\epsilon_{0}\right)^{n}=0
$$

and so, as (2.32) is true for all $n=0,1,2, \ldots$, we can easily be led to (2.19). This completes the proof of the theorem.

\section{References}

[1] Y. Domshlak, Oscillatory properties of linear difference equations with continuous time, Differential Equations Dynam. Systems 1 (1993), no. 4, 311-324.

[2] R. D. Driver, Some harmless delays, Delay and Functional Differential Equations and Their Applications (Proc. Conf., Park City, Utah, 1972), Academic Press, New York, 1972, pp. $103-$ 119.

[3] R. D. Driver, G. Ladas, and P. N. Vlahos, Asymptotic behavior of a linear delay difference equation, Proc. Amer. Math. Soc. 115 (1992), no. 1, 105-112.

[4] R. D. Driver, D. W. Sasser, and M. L. Slater, The equation $x^{\prime}(t)=a x(t)+b x(t-\tau)$ with "small" delay, Amer. Math. Monthly 80 (1973), 990-995.

[5] J. R. Graef and C. Qian, Asymptotic behavior of forced delay equations with periodic coefficients, Commun. Appl. Anal. 2 (1998), no. 4, 551-564.

[6] I.-G. E. Kordonis, N. T. Niyianni, and C. G. Philos, On the behavior of the solutions of scalar first order linear autonomous neutral delay differential equations, Arch. Math. (Basel) 71 (1998), no. 6, 454-464.

[7] I.-G. E. Kordonis and Ch. G. Philos, On the behavior of the solutions for linear autonomous neutral delay difference equations, J. Differ. Equations Appl. 5 (1999), no. 3, 219-233.

[8] I.-G. E. Kordonis, Ch. G. Philos, and I. K. Purnaras, Some results on the behavior of the solutions of a linear delay difference equation with periodic coefficients, Appl. Anal. 69 (1998), no. 1-2, 83-104.

[9] G. Ladas, Recent developments in the oscillation of delay difference equations, Differential Equations (Colorado Springs, Colo, 1989), Lecture Notes in Pure and Appl. Math., vol. 127, Dekker, New York, 1991, pp. 321-332.

[10] G. Ladas, L. Pakula, and Z. Wang, Necessary and sufficient conditions for the oscillation of difference equations, Panamer. Math. J. 2 (1992), no. 1, 17-26.

[11] Ch. G. Philos, Asymptotic behaviour, nonoscillation and stability in periodic first-order linear delay differential equations, Proc. Roy. Soc. Edinburgh Sect. A 128 (1998), no. 6, 1371-1387. 


\section{Difference equations with continuous variable}

[12] Ch. G. Philos and I. K. Purnaras, Periodic first order linear neutral delay differential equations, Appl. Math. Comput. 117 (2001), no. 2-3, 203-222.

[13] M. Pituk, The limits of the solutions of a nonautonomous linear delay difference equation, Comput. Math. Appl. 42 (2001), no. 3-5, 543-550.

[14] Asymptotic behavior of a nonhomogeneous linear recurrence system, J. Math. Anal. Appl. 267 (2002), no. 2, 626-642.

[15] A. N. Sharkovsky, Yu. L. Maĭstrenko, and E. Yu. Romanenko, Difference Equations and Their Applications, Mathematics and Its Applications, vol. 250, Kluwer Academic Publishers, Dordrecht, 1993.

[16] J. H. Shen, Comparison and oscillation results for difference equations with continuous variable, Indian J. Pure Appl. Math. 31 (2000), no. 12, 1633-1642.

[17] J. Yan and F. Zhang, Oscillation for system of delay difference equations, J. Math. Anal. Appl. 230 (1999), no. 1, 223-231.

[18] Y. Zhang, J. Yan, and A. Zhao, Oscillation criteria for a difference equation, Indian J. Pure Appl. Math. 28 (1997), no. 9, 1241-1249.

Ch. G. Philos: Department of Mathematics, University of Ioannina, P.O. Box 1186, 45110 Ioannina, Greece

E-mail address: cphilos@cc.uoi.gr

I. K. Purnaras: Department of Mathematics, University of Ioannina, P.O. Box 1186, 45110 Ioannina, Greece

E-mail address: ipurnara@cc.uoi.gr 